\title{
Efficacy of tuina, cupping and acupuncture therapy on pain, anxiety, depression and quality of life in patients with neck pain: A protocol for systematic review and network meta-analysis
}

\section{Shi-xin Qin}

Anhui University of Traditional Chinese Medicine https://orcid.org/0000-0002-2182-8005

An Li

Anhui University of Traditional Chinese Medicine

Mao-Lin Zhan

The First Affiliated Hospital of Anhui University of Chinese Medicine

\section{Yi-Cheng Wu}

The First Affiliated Hospital of Anhui University of Chinese Medicine

\section{Yong-Hua Zhao}

The First Affiliated Hospital of Anhui University of Chinese Medicine

\section{Zi-Meng Lv}

The First Affiliated Hospital of Anhui University of Chinese Medicine

Yu Zhang ( $\square$ zhangyudyx92@163.com)

The First Affiliated Hospital of Anhui University of Chinese Medicine https://orcid.org/0000-0002-23580904

\section{Protocol}

Keywords: tuina, cupping, acupuncture, pain, anxiety, depression, quality of life, neck pain, systematic review, network meta-analysis

Posted Date: October 21st, 2021

DOl: https://doi.org/10.21203/rs.3.rs-966738/v1

License: (1) This work is licensed under a Creative Commons Attribution 4.0 International License. Read Full License 


\section{Abstract}

Background: Neck pain is a common disorder and is more frequent in females than in males worldwide. Recently, more attention is being paid to precautions for and treatment of neck pain. Current therapeutic methods for neck pain include pharmaceutical, complementary and alternative therapies. Tuina, acupuncture and cupping are effective in the treatment of neck pain, which enrich patients' options. In this study, we aim to evaluate the efficacy of different interventions using randomised controlled trials to identify a prioritised treatment for neck pain.

Methods: We will search five English databases (Web of Science, MEDLINE, EMBASE, OVID and Cochrane Library) and four Chinese databases (CNKI, SinoMed, Wanfang Database and VIP) from database inception to December 2020. Two reviewers will independently perform article screening (title, keywords, abstract and full text); data extraction; risk of bias (RoB) assessment and grading of recommendations assessment, development and evaluation. We will use RevMan 5.3 software to carry out statistical analysis of the RoB and risk ratio to analyse the dichotomous data. Finally, we will use GeMTC V.0.8.1 package of R-3.3.2 software for network meta-analysis based on a Bayesian framework.

Discussion: We will compare these three interventions to determine the most effective therapy for neck pain in terms of improving pain, anxiety, depression and QoL. In this way, we will provide powerful evidence for future clinical practice guidelines for patients with neck pain who want to receive a suitable treatment.

\section{Systematic review registration: PROSPERO CRD42020206853.}

\section{Background}

Neck pain is a common disorder characterised by pain, muscle tension or stiffness localised in the neck, ${ }^{[1]}$ with an average lifetime prevalence of nearly $48 \%$ (depending on age, gender, pain history and poor posture). The global prevalence of neck pain is higher in females than in males. ${ }^{[2]}$ Neck pain not only constitutes a considerable personal burden but also affects patients' families and health system. ${ }^{[3]}$ More attention is now being paid to neck pain precautions and treatment.

Current treatment methods for neck pain include pharmaceutical therapy and non-pharmacological alternatives. Pharmaceuticals include non-steroidal anti-inflammatory drugs (e.g. diclofenac sodium and ibuprofen) and muscle relaxants (e.g. cyclobenzaprine). ${ }^{\text {[2] }}$

Predel et al. ${ }^{[4]}$ evaluated pain on movement, pain at rest and functional neck disability index in patients treated with diclofenac diethylamine (DDEA) or placebo and concluded that DDEA is effective and well tolerated in the treatment of acute neck pain. Hsieh et al. ${ }^{[5]}$ demonstrated that the diclofenac sodium group was superior to the control (menthol) group in reducing pain and improving function, without any significant adverse events (AEs). Borenstein et al. ${ }^{[6]}$ indicated that the 2.5 -mg cyclobenzaprine tid group was not distinctly more effective than the placebo group, but the 5-mg and 10-mg cyclobenzaprine tid 
groups had significantly higher mean efficacy scores than the placebo group. The most common AEs reported in pharmaceutical therapy were somnolence, dry mouth, minor local cutaneous reactions (pruritus and rash) and gastrointestinal effects. ${ }^{[5,7]}$

Complementary and alternative therapies include different treatments such as acupuncture [e.g. electroacupuncture (EA), manual acupuncture (MA) and fire needling (FA)], tuina, cupping, education and exercise. Although acupuncture is used in clinical practice worldwide, it remains controversial for neck pain. ${ }^{[8]}$ A prospective national survey of acupuncture treatment has reported severe tiredness and exhaustion, pain at the needling site and headache as the most common AEs. ${ }^{[9]}$ Although some AEs of acupuncture treatment have been reported, these AEs are much less severe than those of pharmacological treatments and are more easily accepted by patients.

After analysing data from 39 trials with 20,827 patients, Vickers et al. ${ }^{[8]}$ concluded that acupuncture is a positive treatment for neck pain, with treatment effects persisting over time. Zhou et al. ${ }^{[10]}$ and Wang et al. ${ }^{[11]}$ established that the efficacy of warming-needle moxibustion combined with acupotomology or acupuncture combined with tuina is significantly higher than acupuncture alone. However, another study has concluded that the efficacy of the combination of acupuncture and another therapy on neck pain is equivalent to that of acupuncture alone. ${ }^{[12]}$

Currently, it is unknown if tuina, cupping or acupuncture alone or their combined use is best for patients with neck pain. Therefore, it is necessary to determine the best therapy for patients with neck pain. This systematic review and network meta-analysis (NMA) will compare and rank these therapies in terms of treatment efficacy for neck pain. We expect that the outcomes will provide evidence to help determine the best clinical practices and health policies.

\section{Methods}

Our protocol will be conducted according to the Preferred Reporting Items for Systematic Review and Meta-Analysis Protocols(PRISMA-P) statement. ${ }^{[13]}$ We have registered the protocol on PROSPERO (registration number: CRD42020206853).

\section{Eligibility criteria}

Types of studies

This study will only include randomised controlled trials (RCTs), and the study design will strictly follow population, intervention, control, outcome and study design principles. We will not use non-RCTs, animal experiments, human cell or tissue experiments, literature reviews, retrospective studies, case reports, studies with unavailable data and repeatedly published studies.

Types of participants 
We will include patients $>18$ years of age with neck pain according to the International Classification of Diseases (ICD-11, ME84.0) diagnostic guidelines. Inclusion criteria for patients are as follows: neck pain as the main symptom for at least 3 months, pain lasting $>20$ min per time and at least once/month and a neck pain score of $\geq 3$ on the scale of $0-10$, which will be estimated using the visual analogue scale (VAS). Patients who have a specific cause for neck pain (e.g. traumatic injury and whiplash-associated disorder), serious cardiovascular disease or a previous history of neck surgery will be excluded.

Interventions

Studies using tuina therapy, cupping therapy and acupuncture therapy (e.g. EA, MA and FA) alone or in combination as an intervention will be included.

Comparisons

Any comparison is allowed, including comparisons with placebo intervention, no treatment, false stimulation under same conditions or usual care.

Types of outcome measures

Studies reporting one or more of the following outcomes will be included.

Primary outcomes

Primary outcomes will include the VAS and Hamilton Anxiety Scale scores.

Secondary outcomes

Secondary outcomes will include the Oswestry disability index, short form health survey, Pittsburgh sleep quality index and hospital anxiety and depression scale scores.

\section{Search strategy}

We will develop a comprehensive search strategy and search electronic databases established before December 2020, including five English databases (Web of Science, MEDLINE, EMBASE, OVID and Cochrane Library) and four Chinese databases (CNKI, SinoMed, Wanfang Database and VIP) without language restrictions. In addition, we will include gray references (e.g. conference papers and bibliographies) and academic dissertations to reduce publication bias in our data. (See Table 1) 


\section{Table 1 Search strategy}

\begin{tabular}{|c|c|}
\hline & Diseases terms \\
\hline$\# 1$ & $\begin{array}{l}\text { "Neck Pain*" or "Chronic Neck Pain*" or "Neck Ache" or "Neckache*" or "Neck } \\
\text { Aches" or "Cervical Pain*" or "Cervicalgia*" or "Cervicodynia*" or "Anterior } \\
\text { Cervical Pain*" or "Posterior Cervical Pain*" }\end{array}$ \\
\hline \multirow[t]{2}{*}{$\# 2$} & "Pain*" or "Anxiety" or "Depression" or "Quality of Life" \\
\hline & Interventions of terms \\
\hline \multirow[t]{2}{*}{$\# 3$} & $\begin{array}{l}\text { "Tuina" or "Massage" or "Chiropractic" or "Chiropractic Manipulation" or } \\
\text { "Acupoint Pressure" or "Cupping" or "Cupping Therapy" or "Hijama" or } \\
\text { "Acupuncture" or "Acupuncture Points" or "Acupuncture Therapy" or } \\
\text { "Acupuncture Analgesia" }\end{array}$ \\
\hline & RCTs terms \\
\hline \multirow[t]{2}{*}{ \#4 } & $\begin{array}{l}\text { "Randomised Controlled Trial" or "Controlled Clinical Trial" or "Randomised" or } \\
\text { "Placebo" or "Randomisation" or "Randomisation" or "Randomly Trial or Groups" }\end{array}$ \\
\hline & Combination of terms \\
\hline$\# 5$ & $\# 1$ AND \#2 AND \#3 AND \#4 \\
\hline
\end{tabular}

\section{Selection process}

We will select studies and exclude any duplications with NoteExpress V.3.0 (Beijing Aegean Software Co., Ltd, China.). Two reviewers (S-XQ and $A L$ ) will read the title and abstract of each study independently to exclude studies that clearly do not meet the inclusion criteria. Any discrepancy will be discussed with a third reviewer (YZ). Studies that are deemed relevant by at least one reviewer will undergo a full-text review. Then, two reviewers will read the full text to determine the final included reviews. After completing the screening phase, the two reviewers will use Microsoft Excel 2019 to extract information from the included reviews, including general information (author and publication year), review characteristics (study setting and design, inclusion criteria and intervention type) and AEs. ${ }^{[14]}$ (See Figure 1)

\section{Assessment of RoB}

RoB in the methodological quality of the RCTs will be assessed by two reviewers(S-XQ and $A L$ ) independently using the revised Cochrane RoB tool (RoB 2.0) ${ }^{[15]}$ RoB will be classified as high risk, low risk and unclear. The two reviewers will discuss all differences with each other and with a third reviewer (YZ), if necessary.

\section{Assessment of certainty of evidence}


We will use the GRADE approach for NMA to assess the certainty of evidence. ${ }^{[16]}$ The evidence quality evaluation of primary outcomes can be regarded as four levels: high quality, moderate quality, low quality and very low quality. ${ }^{[17]}$ Evidence quality is generally judged on the basis of RoB, inconsistency, indirectness, inaccuracy and dissemination bias.

\section{Statistical analyses}

Measures of treatment effect

Effect sizes for continuous outcomes will be expressed as mean difference or standardised mean difference with a 95\% confidence interval (CI). For dichotomous outcomes, effect sizes will be expressed as risk ratios with a $95 \% \mathrm{Cl}$. We will handle any data issues that arise according to the method described in the Cochrane handbook. ${ }^{[18]}$ In exceptional cases (i.e. if a less number of RCTs are expressed as negative health outcomes continuously, while most of the RCTs used dichotomous outcomes), outcomes reported as continuous or categorical will be dichotomised. ${ }^{[19]}$

\section{Data synthesis}

Firstly, we will use RevMan 5.3 software to carry out statistical analysis of RoB. We will also conduct a quantitative analysis of the heterogeneity of results through $\mathrm{I}^{2}$. When $\mathrm{P}>0.1$, we will neglect the heterogeneity, but if $\mathrm{P}<0.1$ and $\mathrm{I}^{2}>50 \%$, we will determine the possible reasons from both clinical and methodological perspectives as well as provide an explanation or conduct subgroup analysis.

Second, we will use the risk ratio to analyse dichotomous data. Furthermore, we will calculate the corresponding $95 \% \mathrm{Cl}$ for each parameter between the treatment and control groups.

Third, we will use GeMTC V.0.8.1 package of R-3.3.2 software for NMA based on a Bayesian framework to show meta-analysis using the Markov chain Monte Carlo method. ${ }^{[20]}$ We will use the Brooks-GelmanRubin method to assess convergence. We will perform estimation and inference when the convergence state is stable. We will set the GeMTC package's initial parameters as follows: number of chains, 4 ; initial value, 2.5; step size, 5; number of simulation iterations, 100,000 and number of adjustment iterations, 20,000 . Parameters can be adjusted appropriately according to specific conditions. With the potential scale reduced factor close to 1 , we will determine whether the consistency of the model is reliable. Moreover, a ranking figure of all treatment sessions will be generated if multiple intervention times exist.

Sensitivity and subgroup analyses

If possible, we will conduct sensitivity analysis by only including studies rated as low RoB. We will perform sensitivity analysis to monitor the robustness of the primary decision made in the review process. When considerable heterogeneity is detected in an initial analysis, we will perform a subgroup analysis, if necessary. [21]

\section{Ethics and dissemination}


For NMA, there is no direct data collection from human participants; therefore, no ethical approval is necessary. ${ }^{[22]}$

\section{Safety and AEs}

We will record the number and type of AEs in the included studies.

\section{Discussion}

To the best of our knowledge, this is the first study to directly compare the efficacy of tuina, cupping and acupuncture therapy on pain, anxiety, depression and quality of life (QoL) in patients with neck pain.

Pach et al. ${ }^{[23]}$ concluded that giving tuina treatment six times within 3 weeks was beneficial for patients with chronic neck pain, resulting in relevant pain relief and treatment effects maintained after 12 weeks. They also added that future research should compare tuina to other interventions that are effective for neck pain. MacPherson et al. ${ }^{[24]}$ found out that acupuncture-related lifestyle advice helped patients who had chronic neck pain improve their lifestyle. At the same time, it also enhanced self-efficacy and the ability to use what they had learned. These changes were caused by reductions in pain and disability at

12 months. In addition, Lauche et al. ${ }^{[25]}$ pointed out that dry-cupping treatments appeared to be effective in relieving chronic, non-specific neck pain. Not only subjective measures improved, but also mechanical pain sensitivity differed significantly between the two groups, suggesting that cupping affects functional pain processing.

Although numerous studies for neck pain using tuina, cupping or acupuncture can be found, systematic reviews and NMAs for analysing the efficacy of tuina, cupping and acupuncture therapy for neck pain are still scarce.

Many people experience neck pain and it has become a widespread public health problem. Moreover, neck pain is quite common among younger people, even adolescents. ${ }^{[26,27]}$ Hence, carrying out this systematic review and NMA from available literature to evaluate the efficacy of tuina, cupping and acupuncture therapy on pain, anxiety, depression and QoL in patients with neck pain is important.

We will compare these three interventions to determine the most effective therapy for neck pain in terms of improving pain, anxiety, depression and QoL. In this way, we will provide powerful evidence for future clinical practice guidelines for patients with neck pain who want to receive a suitable treatment.

\section{Abbreviations}

RoB: risk of bias; QoL: quality of life; DDEA: diclofenac diethylamine; Aes: adverse events; EA: electroacupuncture; MA: manual acupuncture; FA: fire needling; NMA: network meta-analysis; PRISMA-P: Preferred Reporting Items for Systematic Review and Meta-Analysis Protocols; RCTs: randomised controlled trials; VAS: visual analogue scale; Cl: confidence interval 


\section{Declarations}

Ethics approval and consent to participate

Not applicable

Consent for publication

Not applicable

Availability of data and materials

Not applicable

Competing interests

The authors declare that they have no competing interests

Funding

This work was financially supported by the Clinical Research Foundation of the First Affiliated Hospital of Anhui University of Chinese Medicine(2020yfyzc30) and Key Research and Development Plan of Anhui Province(1804h08020255).

Authors' contributions

S-XQ and AL jointly wrote the manuscript. They are the co-first-authors of the protocol. S-XQ edited part of the manuscript and finished the final version. AL wrote the data analysis plan. M-LZ, Y-CW and Y-HZ provided critical feedback for the manuscript. Z-ML and $Y Z$ contributed to the conception of the study and designed this project. All authors(S-XQ, AL, M-LZ, Y-CW, Y-HZ, Z-ML and YZ) have read and approved the final manuscript.

Acknowledgements

Not applicable

Author details

${ }^{2}$ Department of Tuina, the First Affiliated Hospital of Anhui University of Chinese Medicine, Hefei, China

${ }^{3}$ Department of Tuina, Anhui Integrated Hospital of Traditional Chinese and Western Medicine, Hefei, China

\section{References}


1. CEDRASCHI MONTICONEMAMBROSINIE C, et al. Cognitive-behavioral Treatment for Subacute and Chronic Neck Pain: A Cochrane Review[J]. Spine (Philadelphia, P. 1976), 2015,40(19): 1495-1504.

2. COHEN, S P. HOOTEN W M. Advances in the diagnosis and management of neck pain[J]. BMJ. 2017;358:j3221.

3. KOLAHI SAFIRIS, HOY A. D, et al. Global, regional, and national burden of neck pain in the general population, 1990-2017: systematic analysis of the Global Burden of Disease Study 2017[J]. BMJ, 2020: $\mathrm{m} 791$.

4. GIANNETTI PREDELHG. B, PABST H, et al. Efficacy and safety of diclofenac diethylamine $1.16 \%$ gel in acute neck pain: a randomized, double-blind, placebo-controlled study[J]. BMC Musculoskelet Disord. 2013;14:250.

5. HONG HSIEHL, CHERN C. S, et al. Efficacy and Side Effects of Diclofenac Patch in Treatment of Patients with Myofascial Pain Syndrome of the Upper Trapezius[J]. J Pain Symptom Manage. 2010;39(1):116-25.

6. KORN BORENSTEINDG. S. Efficacy of a low-dose regimen of cyclobenzaprine hydrochloride in acute skeletal muscle spasm: Results of two placebo-controlled trials[J]. Clinical therapeutics. 2003;25(4):1056-73.

7. SIMON HAROUTIUNIAN D A D A.. Topical NSAID Therapy for Musculoskeletal Pain[J]. Pain Medicine, 2010(2010 Apr;11(4)): 535-549.

8. VICKERS A J, VERTOSICK E A, LEWITH G, et al. Acupuncture for Chronic Pain: Update of an Individual Patient Data Meta-Analysis[J]. The Journal of Pain. 2018;19(5):455-74.

9. MACPHERSON H. Patient reports of adverse events associated with acupuncture treatment: a prospective national survey[J]. Quality and Safety in Health Care, 2004,13(5): 349-355.

10. Zhong-liang ZHOU, Jun-chen ZHU, Wang-jun TANG. ZHENG Bao-zhu. Clinical study on akcpotomye holistic loosening combined with warming needle moxibustion in the treatment of nuchal arthralgia[J]. CJTCMP. 2019;34(08):3854-7.

11. Li-hua WANG, Jing MA, Ai-li WANG. Clinical Effect of Acupuncture-moxibustion Combined with Massage on Cervical Spondylotic Radicular Disease[J]. Chinese General Practise. 2018;21(S2):1846.

12. Biyu LUO, Wenbin FU. Clinical effect of grain-moxibustion combined with acupuncture in treating cervical spondylosis with neck pain[J]. Chinese Acupuncture Moxibustion. 2018;38(09):931-3.

13. MOHER SHAMSEERL, CLARKE D. M, et al. Preferred reporting items for systematic review and metaanalysis protocols (PRISMA-P) 2015: elaboration and explanation[J]. BMJ. 2015;349(jan02 1):g7647.

14. MIAKE-LYE I M PAIGENM. BOOTH M S, et al. Association of Spinal Manipulative Therapy With Clinical Benefit and Harm for Acute Low Back Pain[J]. JAMA. 2017;317(14):1451.

15. STERNE J A C, SAVOVIĆ J, PAGE M J, et al. RoB 2: a revised tool for assessing risk of bias in randomised trials.[Z]. 2019: 366, 14898. 
16. SCHUNEMANN H J PUHANMA, MURAD M H, et al. A GRADE Working Group approach for rating the quality of treatment effect estimates from network meta-analysis[J]. BMJ. 2014;349(sep24 5):g5630.

17. G G A D. UNEMANN. GRADE guidelines: 1. IntroductiondGRADE evidence profiles and summary of findings tables[J]. J Clin Epidemiol. 2011;64:383-94.

18. LI CUMPSTONM, PAGE M T. J, et al. Updated guidance for trusted systematic reviews: a new edition of the Cochrane Handbook for Systematic Reviews of Interventions[J]. Cochrane Database of Systematic Reviews; 2019.

19. ANZURES-CABRERA J, SARPATWARI A, HIGGINS JP. Expressing findings from meta-analyses of continuous outcomes in terms of risks[J]. Stat Med. 2011;30(25):2967-85.

20. CALDWELL D M, ADES A E, HIGGINS JP. Simultaneous comparison of multiple treatments: combining direct and indirect evidence[J]. BMJ. 2005;331(7521):897-900.

21. LI R, JIANG Y, HU R, et al. Effectiveness and safety of tenosynovitis of the long head of the biceps brachii with acupuncture: a protocol for a systematic review and meta-analysis[J]. Trials. 2020;21(1):869.

22. SCHOENE TORBAHNG. D, SCHWINGSHACKL L, et al. Effective SLOPE: EffectS of Lifestyle interventions in Older PEople with obesity: a systematic review and network meta-analysis protocol[J]. BMJ Open. 2020;10(10):e38330.

23. PIPER PACHD, LOTZ M. F, et al. Effectiveness and Cost-Effectiveness of Tuina for Chronic Neck Pain: A Randomized Controlled Trial Comparing Tuina with a No-Intervention Waiting List[J]. The Journal of Alternative Complementary Medicine. 2018;24(3):231-7.

24. ELLIOT MACPHERSONH. B, HOPTON A, et al. Lifestyle Advice and Self-Care Integral to Acupuncture Treatment for Patients with Chronic Neck Pain: Secondary Analysis of Outcomes Within a Randomized Controlled Trial[J]. The Journal of Alternative Complementary Medicine. 2017;23(3):180-7.

25. CRAMER LAUCHER, CHOI H. K, et al. The influence of a series of five dry cupping treatments on pain and mechanical thresholds in patients with chronic non-specific neck pain-a randomised controlled pilot study[J]. BMC Complement Altern Med. 2011;11(1):63.

26. SIVERTSEN B MYRTVEITSM, SKOGEN JC, et al. Adolescent Neck and Shoulder Pain-The Association With Depression, Physical Activity, Screen-Based Activities, and Use of Health Care Services[J]. J Adolesc Health. 2014;55(3):366-72.

27. LI Y, LI S, JIANG J, et al. Effects of yoga on patients with chronic nonspecific neck pain[J]. Medicine. 2019;98(8):e14649.

\section{Figures}




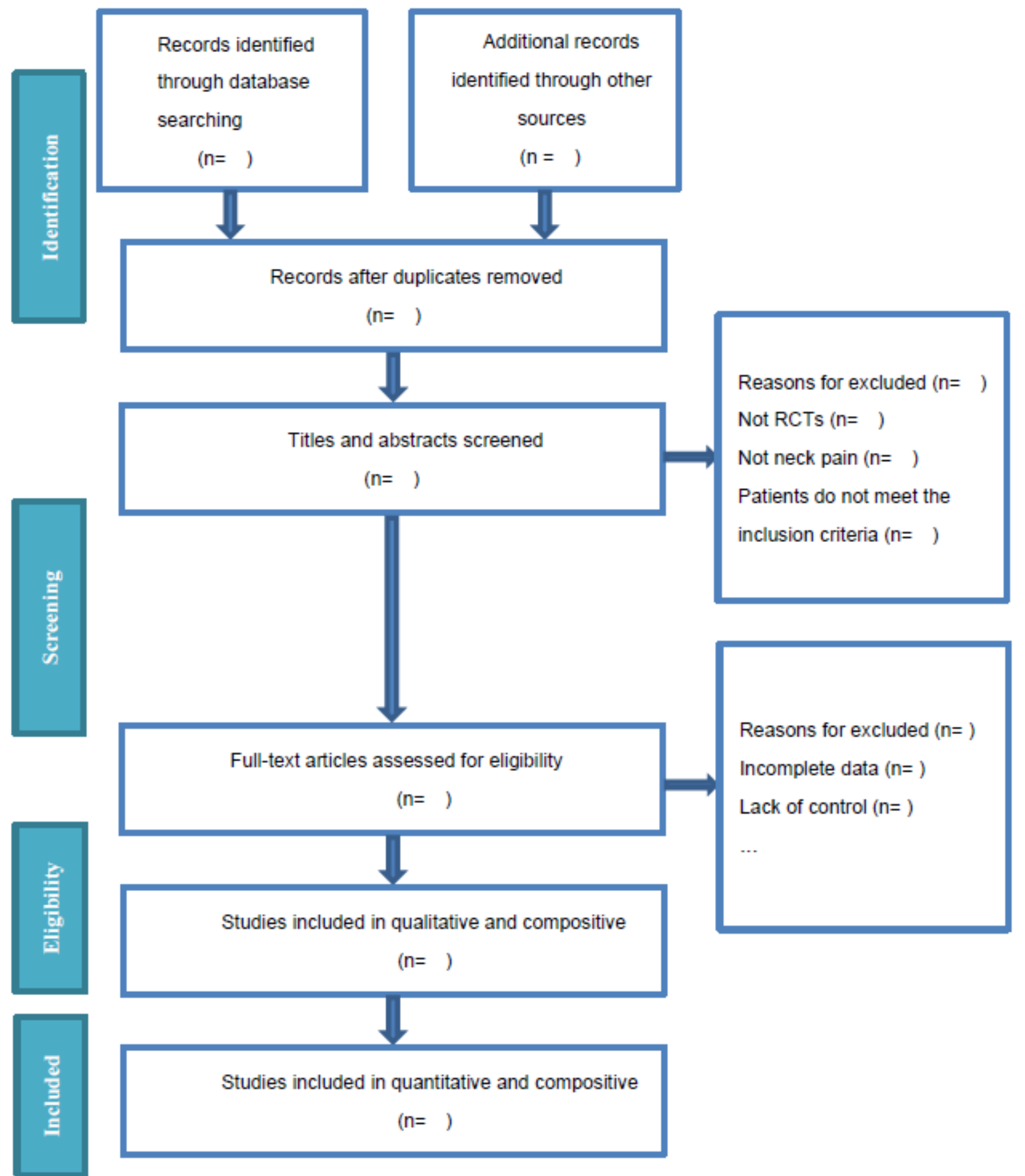

\section{Figure 1}

Flow diagram of the study selection process

\section{Supplementary Files}

This is a list of supplementary files associated with this preprint. Click to download. 
- PRISMAPchecklist.docx 